\title{
A cafeteria diet triggers intestinal inflammation and oxidative stress in obese rats
}

\author{
K. Gil-Cardoso, I. Ginés, M. Pinent, A. Ardévol, X. Terra and M. Blay* \\ Department of Biochemistry and Biotechnology, Mobiofood Research Group, Universitat Rovira $i$ Virgili, 43007 Tarragona, Spain
}

(Submitted 13 September 2016 - Final revision received 23 November 2016 - Accepted 13 December 2016 - First published online 30 January 2017)

\begin{abstract}
The gastrointestinal alterations associated with the consumption of an obesogenic diet, such as inflammation, permeability impairment and oxidative stress, have been poorly explored in both diet-induced obesity (DIO) and genetic obesity. The aim of the present study was to examine the impact of an obesogenic diet on the gut health status of DIO rats in comparison with the Zucker $(f a / f a)$ rat leptin receptordeficient model of genetic obesity over time. For this purpose, female Wistar rats $(n$ 48) were administered a standard or a cafeteria diet (CAF diet) for $12,14.5$ or 17 weeks and were compared with $f a / f a$ Zucker rats fed a standard diet for 10 weeks. Morphometric variables, plasma biochemical parameters, myeloperoxidase (MPO) activity and reactive oxygen species (ROS) levels in the ileum were assessed, as well as the expressions of proinflammatory genes (TNF- $\alpha$ and inducible nitric oxide synthase (iNOS)) and intestinal permeability genes (zonula occludens-1, claudin-1 and occludin). Both the nutritional model and the genetic obesity model showed increased body weight and metabolic alterations at the final time point. An increase in intestinal ROS production and MPO activity was observed in the gastrointestinal tracts of rats fed a CAF diet but not in the genetic obesity model. TNF- $\alpha$ was overexpressed in the ileum of both CAF diet and $f a / f a$ groups, and ileal inflammation was associated with the degree of obesity and metabolic alterations. Interestingly, the 17 -week CAF group and the $f a / f a$ rats exhibited alterations in the expressions of permeability genes. Relevantly, in the hyperlipidic refined sugar diet model of obesity, the responses to chronic energy overload led to time-dependent increases in gut inflammation and oxidative stress.
\end{abstract}

Key words: Gut: Inflammation: Obesity: Cafeteria diet: Oxidative stress

The regular consumption of a high-fat/refined carbohydrate diet such as a typical cafeteria diet (CAF diet) contributes to hyperphagia and obesity as well as other detrimental health consequences linked to obesity, such as insulin resistance and low-grade inflammation ${ }^{(1,2)}$. The hyperphagic and obesogenic effect of a CAF diet is produced via induction of a number of different mechanisms ${ }^{(3)}$. High-fat meals can stimulate innate immune cells and lead to a transient postprandial inflammatory response, altering our immune system and subsequently our inflammatory status ${ }^{(4)}$. Previous studies have pointed to the adipose tissue together with the liver as the main sources and focus of inflammation in obesity ${ }^{(5-8)}$. In addition, the gastrointestinal (GI) tract is another potential target for treating diet-associated inflammation. Among its various functions, the GI tract has the responsibility of informing and protecting the body from diverse chemical structures and metabolising the nutrients required for energy and the plastic functions of human beings ${ }^{(9)}$. In addition, the intestinal epithelium has a conditioning effect on gut homoeostasis, regulating the mucosal immune response and the metabolic activity of the intestine and the entire organism ${ }^{(10)}$. The impact of various dietary constituents including fats and carbohydrates on the GI tract and the microbiota, and subsequent health outcomes in the host, is another exciting and novel area of enquiry. The currently existing knowledge regarding the cellular effects that nutrients exert through the gut supports the possibility of dysregulation of gut homoeostasis by obesogenic diets ${ }^{(11)}$. High-fat/refined carbohydrate diets have been linked to a proinflammatory profile, although this has been poorly studied in the GI tract. Intestinal inflammation is associated with increased myeloperoxidase (MPO) enzyme activity in the ileum; this enzyme is a key component of the $\mathrm{O}_{2}$-dependent microbial activity of phagocytes that, in turn, induces polymorphonuclear neutrophil and macrophage infiltration and results in the production of high levels of proinflammatory cytokines such as TNF- $\alpha^{(11)}$. Cell oxidative stress is another event that is closely linked to tissue inflammation. Nutritional, or dietary, oxidative stress denotes a disturbance in the redox state resulting from excess oxidative load or inadequate nutrient supply, favouring pro-oxidant reactions ${ }^{(12)}$. Reactive oxygen species (ROS)

Abbreviations: CAF diet, cafeteria diet; Fw, forward; GI, gastrointestinal; GO, genetic obesity; HOMA-IR, homeostasis model assessment of insulin resistance; iNOS, isoform of nitric oxide synthase; LepR, leptin receptor; MPO, myeloperoxidase; OCLN, occludin; PC, principal components; ROS, reactive oxygen species; Rv, reverse; TJ, tight junctions; ZO, zonula occludens.

*Corresponding author: M. Blay, fax +34 9775582 32, email mteresa.blay@urv.cat 
production by immune and non-immune cells in response to an obesogenic diet could be a primary event in intestinal inflammation associated with obesity ${ }^{(13)}$. Among all genes, the expression of the inducible isoform of nitric oxide synthase (iNOS) has been widely studied in relation to intestinal inflammation ${ }^{(14,15)}$. During the last decade, it has become increasingly clear that nitric oxide overproduction by iNOS is deleterious to intestinal function ${ }^{(16)}$, thus contributing significantly to GI immunopathology. In addition, gut dysfunction in obesity is also associated with alterations in intestinal permeability. The paracellular pathway is one of the main pathways mediating the transmembrane transfer of specific intestinal components and is perturbed upon disruption of the intestinal barrier. The paracellular pathway is integrated by tight junctions (TJ), a cluster of junctional proteins that selectively regulate the transport of ions, solutes and peptides from the lumen to the intestinal mucosa and bloodstream. Occludin (OCLN) and claudin family members and junctional adhesion molecules are linked to the actin cytoskeleton by cytoplasmic rafts formed by catenins and zonulin/zonula occludens (ZO)-1, -2 and $-3^{(17)}$. During inflammation, persistent, high circulating levels of inflammatory cytokines, which are often observed in obese patients, may cause impairment of intestinal barrier function by altering the structure and localisation of $\mathrm{TJ}^{(18,19)}$. Altogether, this induces an increase in intestinal permeability, which leads to the leakage of water and proteins into the lumen and to translocation of intraluminal solutes such as bacterial endotoxins into the systemic circulation ${ }^{(20)}$. However, a new path regulating the structure and function of the intestinal epithelial barrier through the binding of the adipocyte-derived hormone leptin to its long form leptin receptor (LepR) was recently identified ${ }^{(21-23)}$. Thus, the disruption of TJ-mediated paracellular transport and the overproduction of ROS, as well as alterations in the immune and inflammatory responses and leptin signalling pathways, could be relevant to the obesity phenotype and have not been fully explored in the context of the intestine.

Taking all this into consideration, we hypothesised that a high-fat diet (HFD), high-sucrose diet, a model of an obesogenic diet, could alter intestinal function and not only cause an increase in nutrient absorption but also activate the inflammatory and oxidative stress responses in the gut and alter barrier permeability in healthy rats. We also hypothesised that these changes would be proportional to the length of the nutritional intervention, with different degrees of intestinal inflammation induced by a HFD. Such effects were compared with a genetic model of obesity, $f a / f a$ rats, which develop increased adiposity, hyperphagia, hyperinsulinaemia, hyperlipidaemia and multiple endocrine abnormalities early in life.

The aim of this study was to examine the impact of an obesogenic diet on gut health and the time course of these changes in healthy Wistar rats under a CAF diet in addition to comparing these changes with LepR-deficient obese $(f a / f a)$ Zucker rats as a genetic obesity (GO) model. Specifically, we aimed to evaluate the time course of the alterations that occur during obesity development: obesity degree and metabolic alterations with respect to gut dysfunction, namely, inflammation, oxidative stress and permeability in dietary intervention $v$. GO.

\section{Methods}

\section{Experimental animal procedures}

A total of forty-eight female Wistar rats weighing $230 \mathrm{~g}$ were purchased from Charles River Laboratories and were housed in animal quarters at $22^{\circ} \mathrm{C}$ with a $12 \mathrm{~h}$ light- $12 \mathrm{~h}$ dark cycle (light from 0800 to 2000 h). After 1 week of adaptation, the animals ( $n$ 6-8/group) were randomly selected to receive either a standard diet (A-04; Panlab) (Control group) or a standard diet plus a cafeteria diet (CAF group) for $12,14.5$ or 17 weeks as a model of a high-fat/ high-sucrose diet. Animals were fed ad libitum with fresh food daily. The cafeteria intervention consisted of bacon, sweets, biscuits with paté, cheese, muffins, carrots and sugared milk, with an overall content of $62.2 \%$ carbohydrate, mostly in the form of simple sugars, $23 \%$ lipid and $12.8 \%$ protein with a mineral-vitamin content of $2 \mathrm{~g} / \mathrm{kg}$ of $\operatorname{diet}^{(24)}$. In addition, a GO study was performed with obese $(f a / f a)$ female Zucker rats $(n 10)$ and heterozygous ( $F a / f a)$ lean counterparts ( $n$ 10) with an initial weight of $210 \mathrm{~g}$; these animals received a standard diet for 10 weeks.

At the end of each study, after an overnight fast, animals were anaesthetised with $50 \mathrm{mg} / \mathrm{kg}$ of sodium pentobarbital and killed. Heparinised blood was collected, and plasma was obtained by centrifugation. The intestine and the different white adipose tissue depots (retroperitoneal white adipose tissue, mesenteric white adipose tissue (MWAT) and periovaric white adipose tissue) were rapidly removed, weighed, frozen in liquid $\mathrm{N}_{2}$ and stored at $-80^{\circ} \mathrm{C}$ until later analysis. All experimental procedures were performed according to the National and Institutional Guidelines for Animal Care and Use that are in place at our university. The Animal Ethics Committee of our university approved all procedures (permission no. 4250).

\section{Morphometric variables}

Body weight was monitored weekly, and the percentage of weight gain was calculated. Adiposity was determined with an adiposity index, which was computed for each rat as previously described $^{(24)}$. These variables together with the MWAT depot weight were evaluated as physiological indicators of the degree of obesity in these animals. Homeostatic Model Assessment for Insulin Resistance (HOMA-IR) was quantified for each animal by using the glucose and insulin levels of each animal.

\section{Biochemical assays}

Plasma glucose and TAG were measured using enzymatic colorimetric kits (QCA), and insulin levels were analysed with a rat insulin ELISA kit (Mercodia). The manufacturer's protocol was followed in all cases.

\section{Quantification of myeloperoxidase activity and reactive oxygen species in the ileum}

Tissue samples were homogenised with a Tissue Lyser LT system (Qiagen) in 50-mm-potassium phosphate buffer (Panreac). An aliquot of the homogenate was stored for the subsequent measurement of ROS. The rest of the homogenate was centrifuged at $15000 \mathrm{~g}$ for $15 \mathrm{~min}$ at $4^{\circ} \mathrm{C}$, and the resulting supernatant was discarded. The pellet was then homogenised 
with hexadecyltrimethylammonium bromide (Sigma-Aldrich) and 50-mu-potassium phosphate buffer. The homogenate was sonicated $(20 \mathrm{~s})$, subjected to three freeze-thaw cycles and centrifuged at $15000 \boldsymbol{g}$ for $10 \mathrm{~min}$ at $4^{\circ} \mathrm{C}$. For MPO activity determination, we used an adaptation of the Lenoir method ${ }^{(25)}$. The supernatant was mixed into a solution of phosphate buffer, $0.22 \%$ guaiacol (SigmaAldrich) and $0.3 \% \mathrm{H}_{2} \mathrm{O}_{2}$ (Sigma-Aldrich), and absorbance was read at $470 \mathrm{~nm}$. Enzyme activity was defined as the amount of MPO needed to degrade $1 \mu \mathrm{mol}$ of $\mathrm{H}_{2} \mathrm{O}_{2}$ in 1 minute $(\mathrm{U}=\mu \mathrm{mol} /$ min) and was normalised to milligram of total protein content, which was measured using the Bradford method.

For the measurement of ROS levels, samples were mixed with 1-mм-EDTA buffer (Panreac) and centrifuged at $3000 \boldsymbol{g}$ for $5 \mathrm{~min}$ at $4^{\circ} \mathrm{C}$, after which the pellet was discarded. The intracellular ROS was determined using the 2',7'-dichlorofluorescein diacetate (DCFH-DA) method (Sigma-Aldrich). Fluorescence was measured at $\lambda_{\mathrm{ex}}=485 \mathrm{~nm}$ and $\lambda_{\mathrm{em}}=530 \mathrm{~nm}$ on an FLx800 Fluorescence Reader (BioTek).

\section{Tissue RNA extraction and quantitative reverse transcription PCR}

Total RNA was extracted from $50 \mathrm{mg}$ of ileum using Trizol (Ambion) according to the manufacturer's instructions. The gene expression analysis was completed using both Taqman probes and SYBR Green primers. When TaqMan probes were used, complementary DNA (cDNA) was obtained from $1 \mu \mathrm{g}$ of mRNA using the High-Capacity cDNA Reverse Transcription kit (Applied Biosystems) according to the manufacturer's instructions. Quantitative reverse transcription PCR (qRT-PCR) amplification and detection of $\mathrm{TJ}$ genes were completed using TaqMan Universal PCR Master Mix and the corresponding specific TaqMan probes (Applied Biosystems): Rn02116071_s1 for rat ZO-1, Rn00581740_m1 for rat claudin-1 and Rn01420322_g1 for rat OCLN. All the results were normalised to cyclophilin E (PPIA) (Rn00690933_m1).

For the other genes, cDNA $(5 \mathrm{ng} / \mathrm{ml})$ was subjected to qRT-PCR amplification using SYBR Green PCR Master Mix (Applied Biosystems). The forward $(\mathrm{Fw})$ and reverse $(\mathrm{Rv})$ primer sequences used were as follows: TNF-alpha, Fw: 5-CCTCACACTCAGATCATCTTCTC-3, Rv: 5-TTGGTGGTTTGCTACGACGTG-3; (EGF-like module-containing mucin-like bormone receptor-like 1) F4/80 (EGF-like module-containing mucin-like hormone receptorlike 1 or Erm1) Fw: 5-CTTTGGCTATGGGCTCCCAGTC-3, Rv: 5-GCAAGGAGGGCAGAGTTGATCGTG-3; iNOS, Fw: 5-CACCC GAGATGGTCAGGG-3, Rv: 5-CCACTGACACTCCGCACAA-3; and PPIA (Ciclophilin), Fw: 5-CTTCGAGCTGTTTGCAGACAA-3, Rv: 5-AAGTCACCACCCTGGCACATG-3.

Reactions were run on a qRT-PCR system (Applied Biosystems) where the thermal profile settings were $50^{\circ} \mathrm{C}$ for $2 \mathrm{~min}, 95^{\circ} \mathrm{C}$ for $2 \mathrm{~min}$, and then forty cycles of $95^{\circ} \mathrm{C}$ for $15 \mathrm{~s}$ and $60^{\circ} \mathrm{C}$ for $2 \mathrm{~min}$. The relative mRNA expression levels were calculated using the $2^{-\Delta \Delta C_{t}}$ method, where $\Delta C_{t}=C_{t}$ gene of interest $-C_{t}$ cyclophilin and $\Delta \Delta C_{t}=\Delta C_{t}$ treated samples - the mean of $\Delta C_{t}$ control samples.

\section{Statistical analysis}

The power analysis was carried out $a$ priori during the design stage of the study. For a Student's $t$ test to compare two groups of experimental animals, we calculated the sample size needed in each group to obtain a power of $0 \cdot 80$, when the effect size is moderate $(0.3)$ and a significance level of 0.05 is used. The resulting sample size was six to eight rats depending on the variables analysed.

Results are expressed as means with their standard errors. Effects were assessed using Student's $t$ test to compare either control $v$. CAF groups for each cafeteria diet intervention or obese $f a / f a v . F a / f a$ for the genetic study. $P$ values $<0.05$ were considered statistically significant. These calculations were performed using IBM-SPSS 22.0 software.

Pearson's correlation coefficient was used to test for correlations between the variables evaluated. Unsupervised principal component analysis (PCA) was performed with XLSTAT 2015.5 (Addinsoft) to assess relationships between the expressions of inflammatory markers, TJ proteins, oxidative stress genes in the ileum, and morphometric and metabolic variables. Variables included in the PCA were selected to obtain a Kaiser-MeyerOlkin (KMO) index $>0.5$ as a measure of sampling adequacy. After data scaling, the analysis was based on the correlation matrix, and principal components (PC) were considered significant if they contributed $>5 \%$ to the total variance.

\section{Results}

Increased body weight and metabolic alterations are observed in nutritional and genetic obesity models

After 12, 14.5 or 17 weeks of consuming the CAF diet, rats showed statistically significant increases in body weight relative to controls fed a standard diet. All the CAF groups also presented a significantly higher percentage of body weight gain (Fig. 1), adiposity and mesenteric fat mass compared with the control animals, with this increase being more dramatic at 17 weeks (Table 1). Regarding the genetic model, all the previously mentioned parameters were significantly higher in $f a / f a$

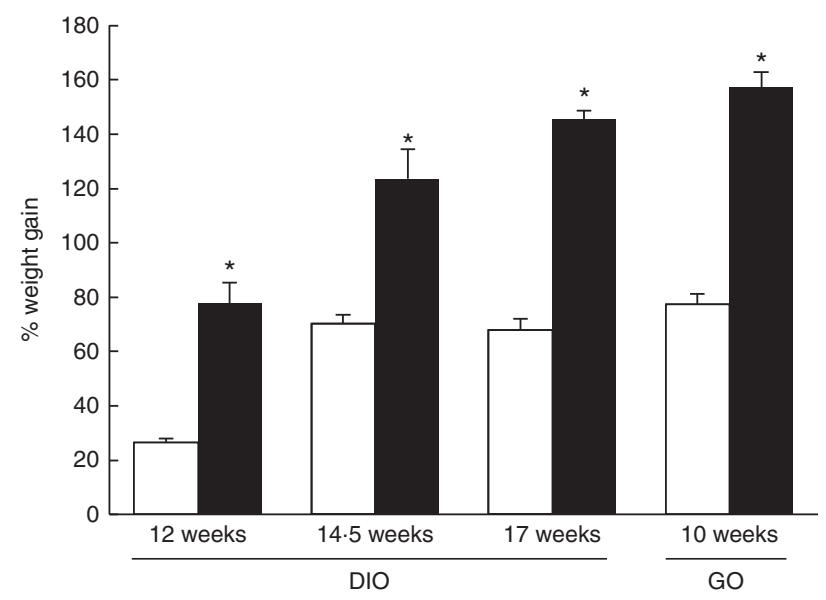

Fig. 1. Weight gain during the different periods of cafeteria diet consumption and in a rat genetic model of obesity. Values are means, with their standard errors. $n 6-10$ in each experimental group. Control $(\square)$ : rats fed a standard diet or lean ( $\mathrm{Fa} / \mathrm{fa}$ ) rats; Obese $(\square)$ : rats fed a cafeteria diet (diet-induced obesity (DIO)) or obese ( $\mathrm{fa} / \mathrm{fa}$ ) rats (genetic obesity (GO)). The statistical significance of these differences was evaluated using Student's $t$ test. * $P$ values $<0.05$, control $v$. obese at the same time point. 
Table 1. Morphometric and metabolic parameters of each experimental group $(n 6-10) \dagger$ (Mean values with their standard errors)

\begin{tabular}{|c|c|c|c|c|c|c|c|c|c|c|c|c|c|c|c|c|}
\hline \multirow[b]{4}{*}{ Measurements } & \multicolumn{12}{|c|}{ Diet intervention duration } & & & & \\
\hline & \multicolumn{4}{|c|}{12 weeks } & \multicolumn{4}{|c|}{14.5 weeks } & \multicolumn{4}{|c|}{17 weeks } & \multicolumn{4}{|c|}{ Genetic obesity (10 weeks) } \\
\hline & \multicolumn{2}{|c|}{ Control } & \multicolumn{2}{|c|}{$\mathrm{CAF}$} & \multicolumn{2}{|c|}{ Control } & \multicolumn{2}{|c|}{ CAF } & \multicolumn{2}{|c|}{ Control } & \multicolumn{2}{|c|}{ CAF } & \multicolumn{2}{|c|}{ Control (Fa/fa) } & \multicolumn{2}{|c|}{ Obese (fa/fa) } \\
\hline & Mean & SEM & Mean & SEM & Mean & SEM & Mean & SEM & Mean & SEM & Mean & SEM & Mean & SEM & Mean & SEM \\
\hline Body weight (g) & $236 \cdot 4$ & $2 \cdot 7$ & $332 \cdot 6^{\star \star}$ & $15 \cdot 2$ & $281 \cdot 0$ & 8.8 & $393.8^{*}$ & $26 \cdot 9$ & 274.5 & $10 \cdot 0$ & $430 \cdot 2^{\star \star}$ & $18 \cdot 0$ & $218 \cdot 1$ & 4.2 & $399 \cdot 1^{\star \star}$ & 8.7 \\
\hline Weight gain (\%) & $26 \cdot 5$ & 1.4 & $77 \cdot 5^{\star \star}$ & 7.9 & $70 \cdot 2$ & $3 \cdot 1$ & $123 \cdot 5^{\star}$ & $10 \cdot 9$ & $67 \cdot 7$ & 4.4 & $145 \cdot 2^{\star *}$ & $3 \cdot 2$ & 77.4 & 3.6 & $157 \cdot 0^{\star \star}$ & $5 \cdot 6$ \\
\hline Mesenteric fat depot $(\mathrm{g})$ & 3.4 & 0.3 & $18 \cdot 3^{\star}$ & 3.9 & 4.7 & 0.5 & $15 \cdot 7^{*}$ & $3 \cdot 1$ & 3.9 & 0.4 & $20 \cdot 2^{*}$ & $2 \cdot 6$ & 1.8 & 0.1 & $7 \cdot 1^{\star \star}$ & 0.3 \\
\hline Adiposity (\%) & 4.7 & 0.4 & $14 \cdot 4^{\star \star}$ & 1.4 & $7 \cdot 0$ & 0.9 & $14 \cdot 5^{\star \star}$ & 0.9 & $6 \cdot 4$ & 0.4 & $15 \cdot 7^{\star *}$ & 0.7 & $2 \cdot 7$ & 0.2 & $6 \cdot 3^{* *}$ & 0.2 \\
\hline Insulin (mU/l) & $27 \cdot 8$ & $7 \cdot 6$ & 63.2 & $16 \cdot 4$ & 23.9 & $5 \cdot 2$ & $56 \cdot 7$ & 21.9 & $25 \cdot 8$ & $5 \cdot 3$ & $134.4^{*}$ & 19.8 & 24.2 & 4.1 & $263 \cdot 3^{\star \star}$ & 23.9 \\
\hline Glucose (mM) & $7 \cdot 1$ & 0.9 & 8.8 & $2 \cdot 1$ & 8.0 & 0.7 & 7.5 & 4.5 & $7 \cdot 0$ & 0.5 & $8 \cdot 6^{*}$ & 0.9 & $7 \cdot 8$ & $2 \cdot 6$ & $9 \cdot 7$ & 1.5 \\
\hline HOMA-IR & $9 \cdot 0$ & $2 \cdot 7$ & $20 \cdot 7$ & $6 \cdot 5$ & $8 \cdot 7$ & $2 \cdot 2$ & 19.6 & $7 \cdot 8$ & 8.0 & $1 \cdot 6$ & $52 \cdot 1^{*}$ & $8 \cdot 6$ & $8 \cdot 7$ & 1.8 & $111 \cdot 0^{\star \star}$ & $7 \cdot 3$ \\
\hline TAG (mM) & 0.4 & 0.1 & $1 \cdot 0^{*}$ & 0.4 & 1.2 & 0.3 & $2 \cdot 2^{*}$ & 0.4 & $\mathrm{~N} / \mathrm{t}$ & & $\mathrm{N} / \mathrm{A}$ & & 1.4 & 0.9 & $3 \cdot 7^{\star \star}$ & 1.36 \\
\hline
\end{tabular}

Control, rats fed a standard diet or lean ( Fa/fa) rats; CAF, cafeteria diet; Obese, obese (fa/fa) rats; HOMA-IR, homeostasis model assessment of insulin resistance; N/A, not available. The statistical significance was evaluated using Student's $t$ test. ${ }^{\star} P$ values $<0.05,{ }^{\star *} P$ values $<0.001$ were considered statistically significant.

† Each group was compared with its control.

obese rats than the Fa/fa control group. The percentage of body weight gained by $f a / f a$ animals after 10 weeks was higher than that gained by the CAF group after 17 weeks. In contrast, the adiposity degree was lower in $f a / f a$ animals, as was mesenteric fat weight (Table 1).

To characterise the obesity models, we also evaluated the glucidic and lipidic profiles. Although all the CAF groups presented higher glucose, insulin and homeostasis model assessment (HOMA) index levels, the differences were only statistically significant after 17 weeks of dietary intervention (Table 1). Regarding the presence of hypertriacylglycerolaemia, TAG levels were higher after 12 and 14.5 weeks of CAF diet; unfortunately, we were not able to evaluate these levels at 17 weeks. All these parameters were altered in GO $f a / f a$ animals, which developed spontaneous hyperglycaemia, hyperinsulinaemia and hypertriacylglycerolaemia after 10 weeks (Table 1).

\section{Intestinal reactive oxygen species production and}

myeloperoxidase activity increased after consumption of a cafeteria diet but not in genetic obesity

We measured ROS levels and MPO activity in the ileum to evaluate the intestinal damage after the nutritional intervention and in the genetic model. As shown in Fig. 2(a), ROS levels increased in accordance with the duration of the dietary intervention, becoming statistically significant at 17 weeks. Notably, the enhancement in MPO activity followed the same pattern and was significantly increased in all cafeteria groups compared with the controls at each time point (Fig. 2(b)). However, no differences were observed when we measured these parameters in genetically obese rats.

Ileal reactive oxygen species levels and myeloperoxidase activity correlate with morphometric and metabolic characteristics after 17 weeks of cafeteria diet consumption

The Pearson's correlation test was used to evaluate for potential associations between ileal ROS or MPO and body weight measures in control and CAF groups. Ileal ROS levels and MPO activity exhibited strong and significant correlations with adiposity, weight gain and mesenteric fat weight in the 17-week group of animals. In addition, MPO activity was also strongly correlated with body weight after 17 weeks (Table 2).

Similar results were obtained when we analysed the relationship between ileal ROS and MPO and the metabolic parameters evaluated. As shown in Table 2, some statistically significant correlations were observed after 17 weeks of dietary intervention and in controls, with ROS levels and MPO activity showing positive associations with insulin resistance indicators. However, no correlations between ROS or MPO and morphometric and metabolic parameters were observed in the genetic model (Table 2).

TNF- $\alpha$ is over-expressed in the ileum of cafeteria diet and $\mathrm{fa} / \mathrm{fa}$ groups

To evaluate the inflammatory state in the ileum, we analysed the expressions of the proinflammatory markers TNF- $\alpha$ and iNOS and the expression of $F 4 / 80$, a murine macrophage infiltration marker. Fig. 3 shows the expressions of these genes in the rat ileum after 14.5 and 17 weeks of the CAF diet. The expressions of these markers increased over both time periods, with statistical significance only being reached for TNF-alpha after 14.5 weeks (Fig. 3(b)). Furthermore, TNF-alpha and iNOS expressions were positively correlated ( $r$ 0.499, $P=0.030$ ). TNF-alpha expression was also correlated with the macrophage marker $F 4 / 80$ ( $r 0.491$, $P=0.028$ ), and $i N O S$ expression also appeared to correlate with the levels of this marker $(r 0.417, P=0.068)$. Interestingly, TNF-alpha expression was significantly increased in the $f a / f a$ group (Fig. 3 (b)), but no changes were observed in the rest of genes.

\section{Ileal inflammation is associated with degree of obesity and} metabolic alterations

As inflammation has been linked to obesity and associated insulin resistance, we tested for correlations between ileal $T N F-\alpha, i N O S$ and $F 4 / 80$ expressions and the metabolic and 
(a)

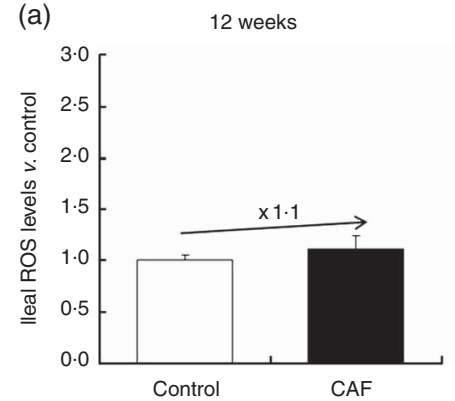

(b)

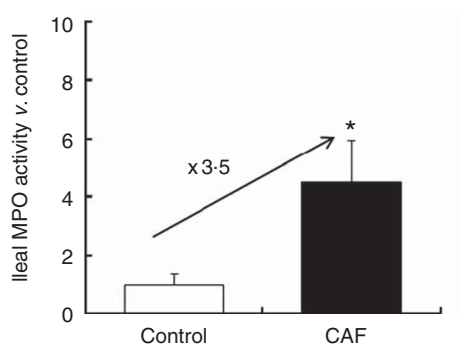

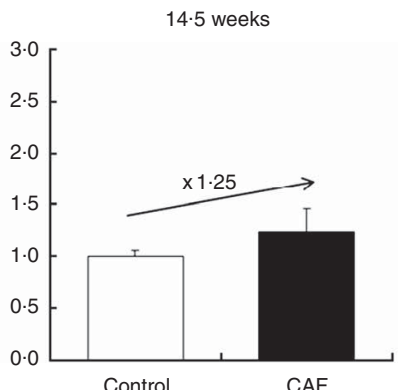

14.5 weeks

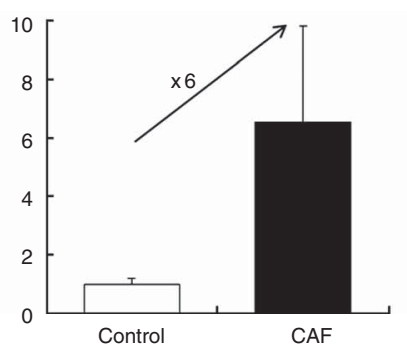

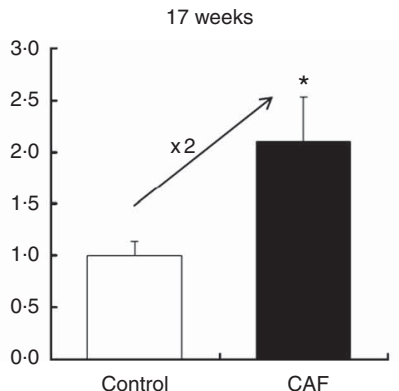

17 weeks

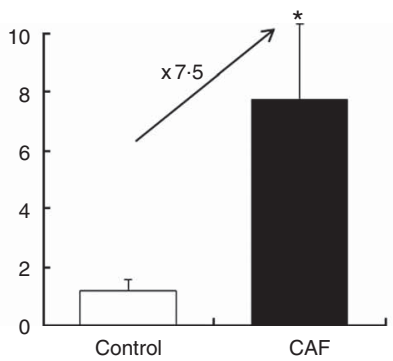

GO

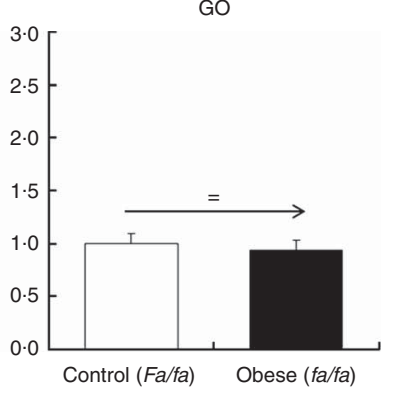

GO

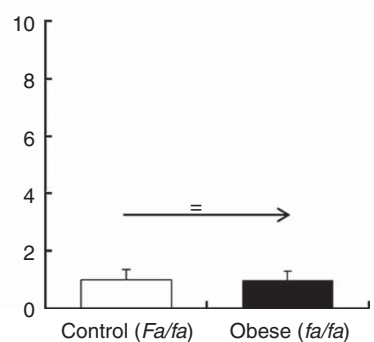

Fig. 2. Ileal reactive oxygen species (ROS) levels (a) and myeloperoxidase (MPO) activity (b) during the different periods of cafeteria or standard diet consumption and in the rat genetic model of obesity. Values are means, with their standard errors. $n 6-10$ in each experimental group. Control: rats fed a standard diet or lean (Fa/fa) rats; CAF: rats fed a cafeteria diet (diet-induced obesity); and Obese: obese (fa/fa) rats (genetic obesity (GO)). The statistical significance of these differences was evaluated using Student's $t$ test. * $P$ values $<0.05$, control $v$. CAF or obese at the same time point.

Table 2. Correlation coefficients for ileal reactive oxygen species (ROS) levels or myeloperoxidase (MPO) activity and morphometric characteristics in the control and cafeteria groups at different time points and in the genetic obesity (GO) model†

\begin{tabular}{|c|c|c|c|c|}
\hline & \multicolumn{3}{|c|}{ Diet intervention duration } & \multirow{2}{*}{$\frac{\mathrm{GO}}{10 \text { weeks }}$} \\
\hline & 12 weeks & 14.5 weeks & 17 weeks & \\
\hline \multicolumn{5}{|l|}{ ROS levels } \\
\hline Body weight (g) & 0.277 & 0.620 & 0.570 & -0.141 \\
\hline Weight gain (\%) & 0.320 & 0.139 & $0.592^{*}$ & -0.014 \\
\hline Mesenteric fat depot $(\mathrm{g})$ & 0.304 & $0.795^{\star}$ & $0.688^{*}$ & -0.194 \\
\hline Adiposity (\%) & 0.381 & 0.472 & $0.662^{*}$ & -0.145 \\
\hline \multicolumn{5}{|l|}{ MPO activity } \\
\hline Body weight (g) & 0.344 & 0.679 & $0.798^{*}$ & 0.042 \\
\hline Weight gain (\%) & 0.350 & 0.295 & $0.740^{*}$ & 0.078 \\
\hline Mesenteric fat depot $(\mathrm{g})$ & 0.174 & 0.605 & $0 \cdot 812^{*}$ & -0.117 \\
\hline Adiposity (\%) & 0.501 & 0.437 & $0 \cdot 778^{*}$ & -0.085 \\
\hline \multicolumn{5}{|l|}{ ROS levels } \\
\hline Insulin (mU/l) & 0.275 & 0.504 & $0.649^{*}$ & -0.159 \\
\hline Glucose (mM) & 0.030 & 0.147 & 0.413 & 0.228 \\
\hline HOMA-IR & 0.163 & 0.531 & $0.603^{*}$ & -0.122 \\
\hline TAG (mM) & 0.267 & 0.284 & N/A & -0.100 \\
\hline \multicolumn{5}{|l|}{ MPO activity } \\
\hline Insulin (mU/I) & -0.036 & 0.437 & $0.757^{*}$ & -0.178 \\
\hline Glucose (mM) & -0.010 & 0.169 & $0.814^{*}$ & 0.403 \\
\hline HOMA-IR & -0.042 & 0.425 & $0.792^{*}$ & -0.099 \\
\hline TAG (mM) & 0.507 & 0.667 & N/A & 0.129 \\
\hline
\end{tabular}

HOMA-IR, homeostasis model assessment of insulin resistance; N/A, not available. ${ }^{*} P$ values $<0.05$ were considered statistically significant.

† The number of animals ( $n$ 12-20) used in each time period in the control and cafeteria groups together is shown.

morphometric characteristics measured over two time periods, 14.5 and 17 weeks.

As shown in Table 3, at 14.5 weeks, TNF- $\alpha$ expression was positively associated with measures of body weight and adiposity, as well as with TAG and insulin levels. Importantly, iNOS expression was also correlated with glucose metabolism parameters and TAG levels. F4/80 also correlated positively with TAG levels at 14.5 weeks. At 17 weeks of the dietary intervention (Table 3), expression was positively associated with adiposity, and iNOS expression correlated positively with glucose levels.

On the other hand, as shown in the Table 3, TNF- $\alpha$ expression in $f a / f a$ obese animals was positively associated with the following morphometric parameters: body weight, mesenteric fat depot and adiposity, and TAG.

The main correlations found with dietary intervention periods of 14.5 and 17 weeks were maintained even after adjustment for body weight (see Supplementary Table S1). In the GO model, when body weight adjustment was applied, we found positive associations of macrophage infiltration (F4/80 expression) with adiposity. In addition, the correlations found with TAG levels persisted. However, other associations were not present after body weight adjustment (Supplementary Table S1).

\section{Tight junctions gene expression is altered in the 17-week cafeteria diet and fa/fa groups}

The expressions of TJ element genes were evaluated as an approach to measure ileal permeability. The results indicate that the expressions of ZO-1 and claudin-1 were strongly reduced after 17 weeks of the CAF diet (Fig. 3(d) and (f), respectively). In addition, $Z O-1$ gene expression was negatively associated with insulin levels (Table 3), and more interestingly ZO-1 maintained a strong negative correlation with homeostasis model assessment of insulin resistance (HOMA-IR) even after 

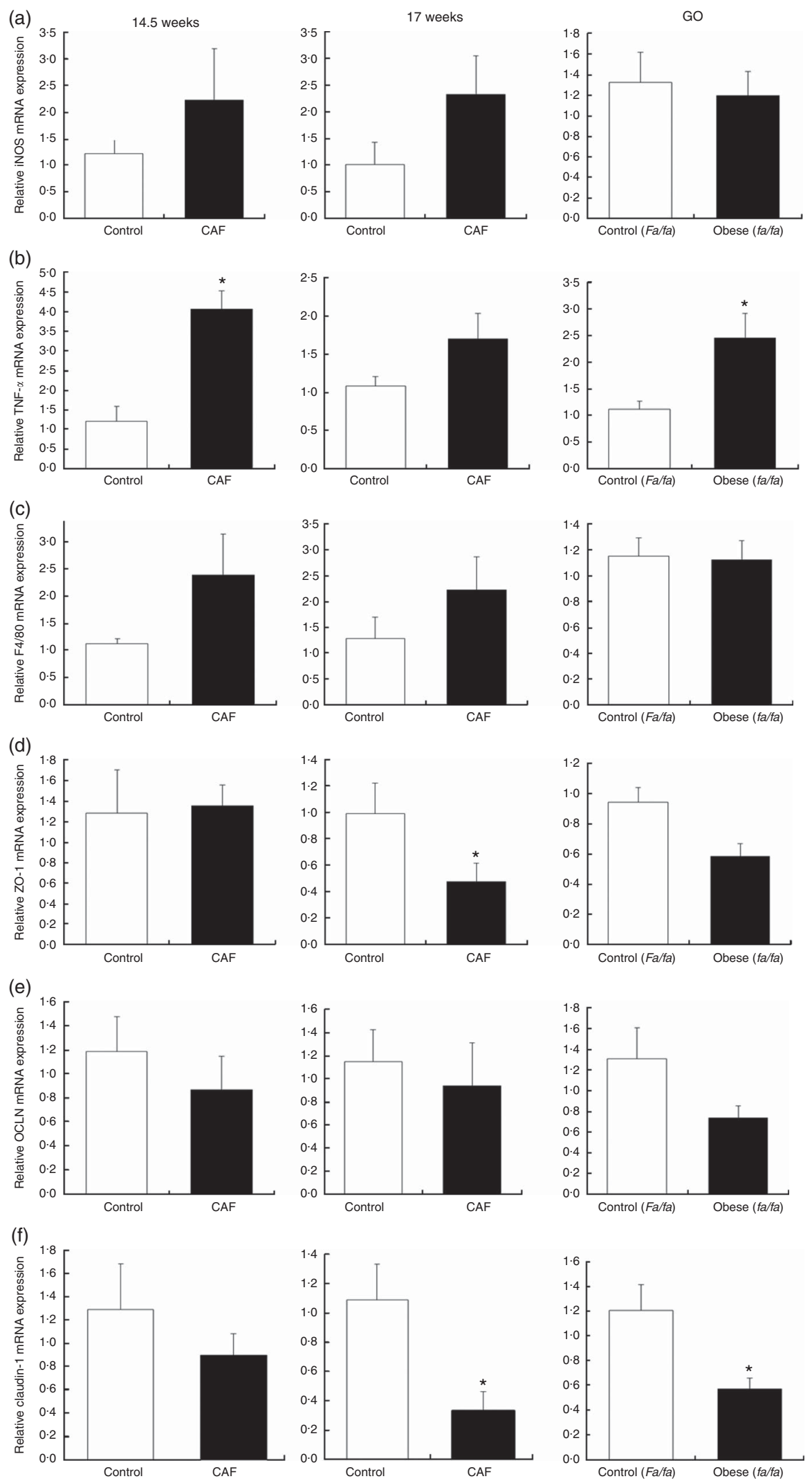

Fig. 3. Ileal mRNA expressions of isoform of nitric oxide synthase (iNOS) (a), TNF- $\alpha$ (b), EGF-like module-containing, mucin-like hormone receptor 1 (F4/80) (c), zonula occludens-1 (ZO-1) (d), occludin (OCLN) (e) and claudin-1 (f) during the different periods of cafeteria or standard diet consumption and in the rat genetic model of obesity. Values are means, with their standard errors. $n 6-10$ in each experimental group. Control: rats fed a standard diet or lean (Fa/fa) rats; CAF: rats fed a cafeteria diet (diet-induced obesity); and Obese: obese (fa/fa) rats (genetic obesity (GO)). The statistical significance of these differences was evaluated using Student's $t$ test. ${ }^{*} P$ values $<0.05$, control $v$. CAF or obese at the same time point. 
Table 3. Correlation coefficients for ileal gene expression and metabolic and morphometric characteristics in rats subjected to 14.5 and 17 weeks of dietary intervention and in a rat genetic model of obesity and respective controls

\begin{tabular}{|c|c|c|c|c|c|c|}
\hline & iNOS & $T N F-a$ & $F 4 / 80$ & $z O-1$ & OCLN & Claudin-1 \\
\hline \multicolumn{7}{|c|}{ Diet intervention: 14.5 weeks ( $n 12$ animals) } \\
\hline \multicolumn{7}{|c|}{ Morphometric variables } \\
\hline Body weight (g) & 0.463 & $0.825^{\star *}$ & 0.254 & 0.028 & -0.415 & -0.164 \\
\hline Weight gain $(\%)$ & 0.193 & $0.946^{\star \star}$ & 0.478 & 0.244 & -0.372 & -0.081 \\
\hline Mesenteric fat depot $(\mathrm{g})$ & 0.304 & 0.697 & $0 \cdot 152$ & -0.031 & -0.457 & -0.199 \\
\hline Adiposity (\%) & 0.384 & $0.890^{* *}$ & 0.417 & 0.173 & -0.275 & -0.182 \\
\hline \multicolumn{7}{|l|}{ Metabolic variables } \\
\hline Insulin (mU/I) & $0.768^{\star *}$ & $0.675^{*}$ & $0 \cdot 192$ & -0.159 & -0.113 & -0.103 \\
\hline Glucose (mM) & 0.076 & -0.183 & -0.301 & -0.300 & 0.206 & -0.172 \\
\hline HOMA-IR & $0.749^{*}$ & 0.638 & 0.151 & -0.185 & -0.101 & -0.131 \\
\hline TAG $(\mathrm{mM})$ & $0.658^{*}$ & $0.937^{\star *}$ & $0.578^{*}$ & 0.127 & -0.092 & -0.162 \\
\hline \multicolumn{7}{|c|}{ Diet intervention: 17 weeks ( $n 12$ animals) } \\
\hline \multicolumn{7}{|c|}{ Morphometric variables } \\
\hline Body weight $(\mathrm{g})$ & 0.429 & 0.332 & 0.354 & -0.266 & -0.129 & $-0.630^{*}$ \\
\hline Weight gain $(\%)$ & 0.450 & 0.410 & 0.418 & -0.283 & -0.180 & $-0.640^{*}$ \\
\hline Mesenteric fat depot $(\mathrm{g})$ & 0.347 & 0.382 & 0.362 & -0.347 & -0.078 & $-0.632^{*}$ \\
\hline Adiposity (\%) & 0.428 & $0.523^{*}$ & 0.421 & -0.352 & -0.142 & $-0.615^{\star}$ \\
\hline \multicolumn{7}{|l|}{ Metabolic variables } \\
\hline Insulin (mU/l) & 0.286 & 0.391 & 0.171 & $-0.447^{\star}$ & -0.036 & -0.575 \\
\hline Glucose (mM) & $0.684^{*}$ & 0.274 & -0.056 & -0.454 & 0.072 & -0.531 \\
\hline HOMA-IR & 0.357 & 0.370 & 0.102 & -0.430 & 0.078 & -0.559 \\
\hline TAG $(\mathrm{mM})$ & $\mathrm{N} / \mathrm{A}$ & $\mathrm{N} / \mathrm{A}$ & $\mathrm{N} / \mathrm{A}$ & $\mathrm{N} / \mathrm{A}$ & $\mathrm{N} / \mathrm{A}$ & $\mathrm{N} / \mathrm{A}$ \\
\hline \multicolumn{7}{|l|}{ Genetic obesity ( $n 20$ animals) } \\
\hline \multicolumn{7}{|l|}{ Morphometric variables } \\
\hline Body weight $(\mathrm{g})$ & -0.116 & $0.514^{*}$ & -0.128 & $-0.614^{*}$ & -0.384 & $-0.588^{*}$ \\
\hline Weight gain $(\%)$ & 0.008 & 0.426 & -0.097 & $-0.529^{*}$ & -0.294 & $-0.580^{\star}$ \\
\hline Mesenteric fat depot (g) & 0.002 & $0.511^{*}$ & 0.033 & $-0.508^{*}$ & -0.370 & $-0.587^{*}$ \\
\hline Adiposity (\%) & 0.011 & $0.491^{*}$ & 0.080 & -0.465 & -0.320 & $-0.649^{*}$ \\
\hline \multicolumn{7}{|l|}{ Metabolic variables } \\
\hline Insulin (mU/l) & 0.075 & 0.410 & 0.104 & -0.422 & -0.366 & $-0.521^{*}$ \\
\hline Glucose (mM) & -0.281 & 0.060 & -0.367 & -0.196 & -0.252 & -0.194 \\
\hline HOMA-IR & 0.002 & 0.400 & 0.010 & $-0.507^{*}$ & -0.404 & $-0.533^{*}$ \\
\hline TAG $(\mathrm{mM})^{\star}$ & -0.176 & $0.699^{*}$ & -0.209 & $-0.744^{*}$ & -0.365 & $-0.704^{\star}$ \\
\hline
\end{tabular}

iNOS, isoform of nitric oxide synthase; F4/80, EGF-like module-containing, mucin-like hormone receptor 1; ZO-1, zonula occludens-1; OCLN, occludin; HOMA-IR, homeostasis model assessment of insulin resistance; N/A, not available.

${ }^{\star} P$ values $<0.05,{ }^{* \star} P$ values $<0.001$ were considered statistically significant.

body weight correction (Supplementary Table S1). However, OCLN expression levels over both time periods were similar to the levels observed in control rats (Fig. 3(e)).

In the case of the GO $f a / f a$ group, $Z O-1$ and claudin-1 were significantly down-regulated in the ileum with respect to the $\mathrm{Fa} / \mathrm{fa}$ control group (Fig. 3(d) and (f)), and OCLN expression showed a negative trend, as shown in Fig. 3(e). ZO-1 and claudin-1 levels were negatively correlated with morphometric parameters, HOMA index and TAG. Claudin-1 was also negatively associated with high insulin levels.

\section{Principal components analysis}

We used a model that reduces a matrix of data to the lowest dimension of the most significant components, or PC, to analyse the overall distribution and associations between the variables evaluated. With this method, the PC retained are generated sequentially, meaning the variance explained by the first component is removed before the second factor is generated to maximally explain the remaining variance in the matrix (this process is continuous with successive components). This tool is useful for identifying various combinations of variables that could reflect possible biological mechanisms, especially in association with various other health outcomes.
Table 4 shows the PCA results for all the variables analysed in the dietary intervention and the GO experiment. The projections in the plane of ileal gene expressions of inflammatory markers, TJ proteins, oxidative stress, and morphometric and metabolic variables defined by the first two PC of the 14.5- and 17-week diet interventions and GO model are shown in Fig. $4(\mathrm{a}-\mathrm{c})$, respectively (left panel). On the other hand, the distribution of animals in the space based on the different variables is represented in the corresponding right panel.

The results obtained after 14.5 weeks of CAF intervention indicate that the first two PC explain $77.86 \%$ of the total variance. The first and the second PC are responsible for 45.49 and $32.37 \%$ of the total variance, respectively. Overall, PC1 was clearly characterised by the following variables: body weight and mesenteric weight, insulin levels and HOMA index, ileal MPO activity and ROS levels, and ileal iNOS mRNA levels (Table 4). As shown in Fig. 4(a), all these variables are grouped in the right side of the graphic, indicating a positive association between the degree of obesity in these animals and insulin pathway metabolic alterations and intestinal inflammation. On the other hand, PC2 is characterised by the percentage of adiposity, weight gain, plasma leptin levels and ileal $T N F-\alpha$ gene expression, which are located in the upper part of the graphic, and by the glucose levels, which are located in the 
Table 4. Loadings for the first two principal components

\begin{tabular}{|c|c|c|c|c|c|c|}
\hline \multirow[b]{3}{*}{ Variables } & \multicolumn{4}{|c|}{ Diet intervention duration } & \multirow{2}{*}{\multicolumn{2}{|c|}{$\frac{\mathrm{GO}}{10 \text { weeks }}$}} \\
\hline & \multicolumn{2}{|c|}{14.5 weeks } & \multicolumn{2}{|c|}{17 weeks } & & \\
\hline & D1 & D2 & D1 & D2 & D1 & D2 \\
\hline ROS & 0.605 & 0.123 & 0.352 & 0.658 & -0.225 & 0.529 \\
\hline Weight & 0.827 & 0.536 & 0.728 & 0.637 & 0.959 & 0.142 \\
\hline Mesenteric weight & 0.768 & 0.539 & 0.722 & 0.652 & 0.975 & -0.050 \\
\hline Adiposity & 0.633 & 0.724 & 0.688 & 0.703 & 0.929 & -0.061 \\
\hline Insulin & 0.961 & 0.036 & 0.781 & 0.534 & 0.928 & -0.116 \\
\hline Glucose & 0.406 & -0.779 & 0.914 & 0.246 & 0.425 & 0.655 \\
\hline Leptin & 0.640 & 0.734 & 0.764 & 0.625 & & \\
\hline Weight gain & 0.166 & 0.954 & 0.648 & 0.691 & 0.935 & $0 \cdot 150$ \\
\hline HOMA-IR & 0.971 & -0.009 & 0.826 & 0.480 & 0.958 & 0.009 \\
\hline MPO & 0.633 & 0.283 & 0.835 & 0.284 & -0.112 & 0.787 \\
\hline iNOS mRNA & 0.542 & 0.119 & 0.612 & 0.101 & -0.112 & -0.453 \\
\hline$T N F-a$ mRNA & 0.506 & 0.785 & 0.122 & 0.630 & 0.607 & -0.144 \\
\hline F4/80 mRNA & & & -0.302 & 0.900 & 0.007 & -0.801 \\
\hline$Z O-1$ mRNA & & & -0.651 & 0.144 & -0.673 & -0.054 \\
\hline Claudin-1 mRNA & & & -0.436 & -0.628 & & \\
\hline OCLN mRNA & & & & & -0.481 & -0.266 \\
\hline
\end{tabular}

ROS, reactive oxygen species; HOMA-IR, homeostasis model assessment of insulin resistance; MPO, myeloperoxidase; iNOS, isoform of nitric oxide synthase; F4/80, EGF-like module-containing, mucin-like hormone receptor $1 ; Z O-1$, zonula occludens-1; OCLN, occludin.

lower zone. With respect to animal distribution, PC2 discriminates between the control and the CAF groups. Unlike the control group, the CAF group is located on the top of the graph and shows a higher percentage of adiposity, greater weight gain, higher plasma levels of leptin and higher ileal $T N F-\alpha$ expression than the control group. However, PC1 does not discriminate between these groups.

In the 17-week CAF consumption group (Table 4), PC1 and PC2 explain $76.56 \%$ of the total variance ( 43.80 and $32.76 \%$, respectively). With respect to $\mathrm{PC} 1$, body weight, mesenteric weight, HOMA index, and insulin, glucose and leptin levels, MPO activity and $i N O S$ gene expression in the ileum were negatively associated with ileal $Z O-1$ mRNA levels. In addition, PC2 is described by adiposity, weight gain, ROS levels, and claudin-1, TNF- $\alpha$ and F4/80 mRNA levels. In this context, claudin-1 expression was negatively associated with adiposity, insulin resistance and inflammatory variables. On the basis of the distribution of the animal groups, PC1 and PC2 discriminate between the control and the CAF groups. As expected, most of the individuals in the CAF group are located in the upper right area of the space that is related to diet-induced obesityassociated metabolic alterations, intestinal inflammation and lower levels of the TJ protein (Fig. 4(b)).

Regarding GO model analysis, the first two PC explain $64.48 \%$ of the total variance. The first PC, which is responsible for $47.83 \%$, is characterised by the morphometric variables including body weight, mesenteric weight, weight gain and adiposity, in addition to the following variables - insulin level, HOMA index and ileal TNF- $\alpha$ gene expression - which are all negatively associated with ileal $Z O-1$ and $O C L N$ mRNA levels. In addition, $\mathrm{PC} 2$ is responsible for $16.65 \%$ of the total variance and is composed of ROS levels, MPO activity in the ileum and plasma glucose levels, which are negatively associated with iNOS and F4/80 mRNA levels. Moreover, PC1 discriminates between the control and the CAF groups. The right panel shows that obese animals are located on the right side of the space that is defined by a higher degree of obesity, altered insulin function, disrupted inflammatory markers and the down-regulation of TJ proteins with respect to the control group (Fig. 4(c)).

\section{Discussion}

The GI tract has energetic, immune and barrier functions that serve to protect the body against infection and to absorb nutrients. Specific parts of the GI tract, particularly the ileum of the small intestine, are specialised in both nutrient absorption and immune defence. Thus, the organs and tissues involved in the regulation of the metabolism, in addition to providing the nutrients necessary to trigger and sustain the immune response, contain resident populations of immune cells, suggesting that the immune system is poised to respond to nutrient-derived signals.

It was recently noted that the GI tract, specifically the gut, is an important target organ to be considered in obesity outcome because of the effects of diet on this organ. As expected, the chronic consumption of a high-energy diet for 12, 14.5 or 17 weeks induces a time-dependent increase in body weight that is accompanied by an increase in adiposity. Insulin resistance and hypertriacylglycerolaemia, typical symptoms of the metabolic syndrome, are reached at the 17-week time point. In agreement with the literature ${ }^{(25)}$, the obesity level reached in the genetic model at 10 weeks was comparable with the results obtained after 17 weeks of CAF diet consumption. In fact, Zucker obese rats became hyperglycaemic at 13-15 weeks of age ${ }^{(26)}$, with hyperinsulinaemia and hypertriacylglycerolaemia appearing after $12-14$ weeks of age ${ }^{(27)}$, suggesting a good model of early-onset obesity.

Regarding the status of the gut, our results showed that ROS levels in the ileal fraction of the small intestine were slightly increased during the first few weeks, reaching significance only at 17 weeks. Different epidemiological, animal and clinical studies have associated obesity with redox disruption ${ }^{(28)}$. A high-fat, high-carbohydrate diet and continuous hypernutrition can increase oxidative stress through the activation of intracellular pathways such as the nitrogen oxide pathways, oxidative phosphorylation pathway in the mitochondria, glycoxidation pathway, protein kinase $\mathrm{C}$ pathway and the polyol pathway $^{(12,29-31)}$. However, oxidative stress can be the cause as well as the outcome of obesity. In this study, we demonstrated that an obesogenic diet is able to induce oxidative stress in the ileum at later time points, suggesting that oxidative damage in this tissue is the result of a severe state of obesity. Moreover, no changes were observed in $f a / f a$ Zucker rats in our study, although the degree of obesity reached is comparable with that in the 17-week CAF diet model. This fact reinforces the above idea that the intestinal oxidative stress observed in these animals is mainly associated with the ingestion of a high-fat, high-fructose diet and not just obesity.

In contrast to oxidative stress, inflammation within the ileum, indicated by an increase in MPO activity, was apparent at the first time point and increased to a maximal level at 17 weeks. MPO is a key enzyme in the $\mathrm{O}_{2}$-dependent microbial activity of 
(a)

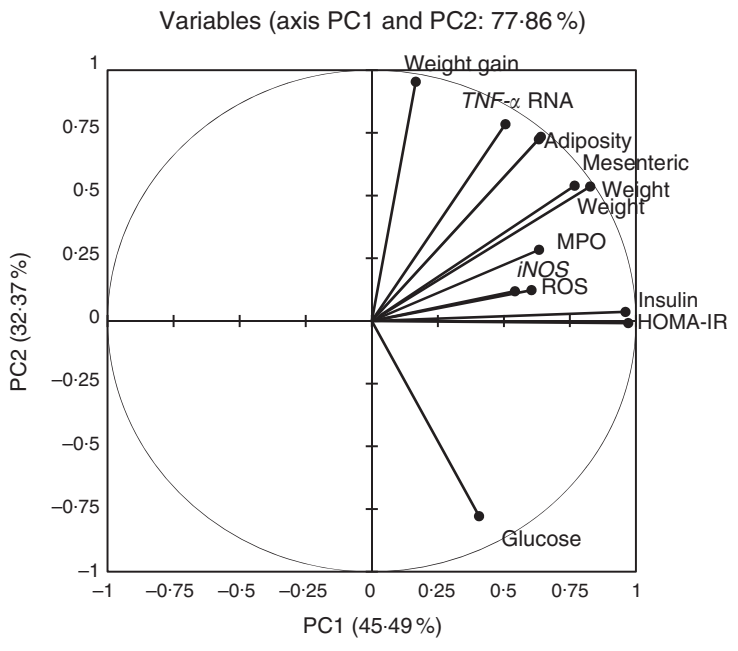

14.5 weeks

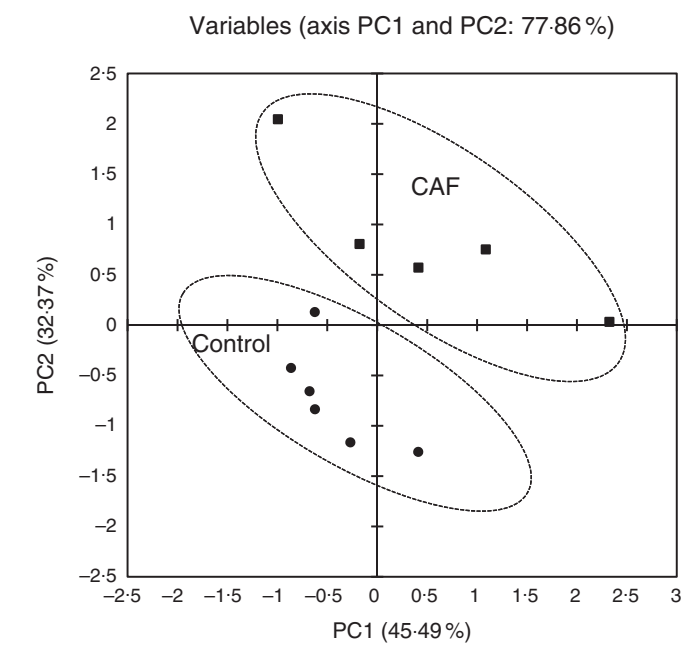

(b)

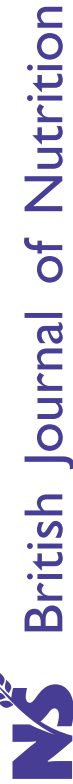

(c)
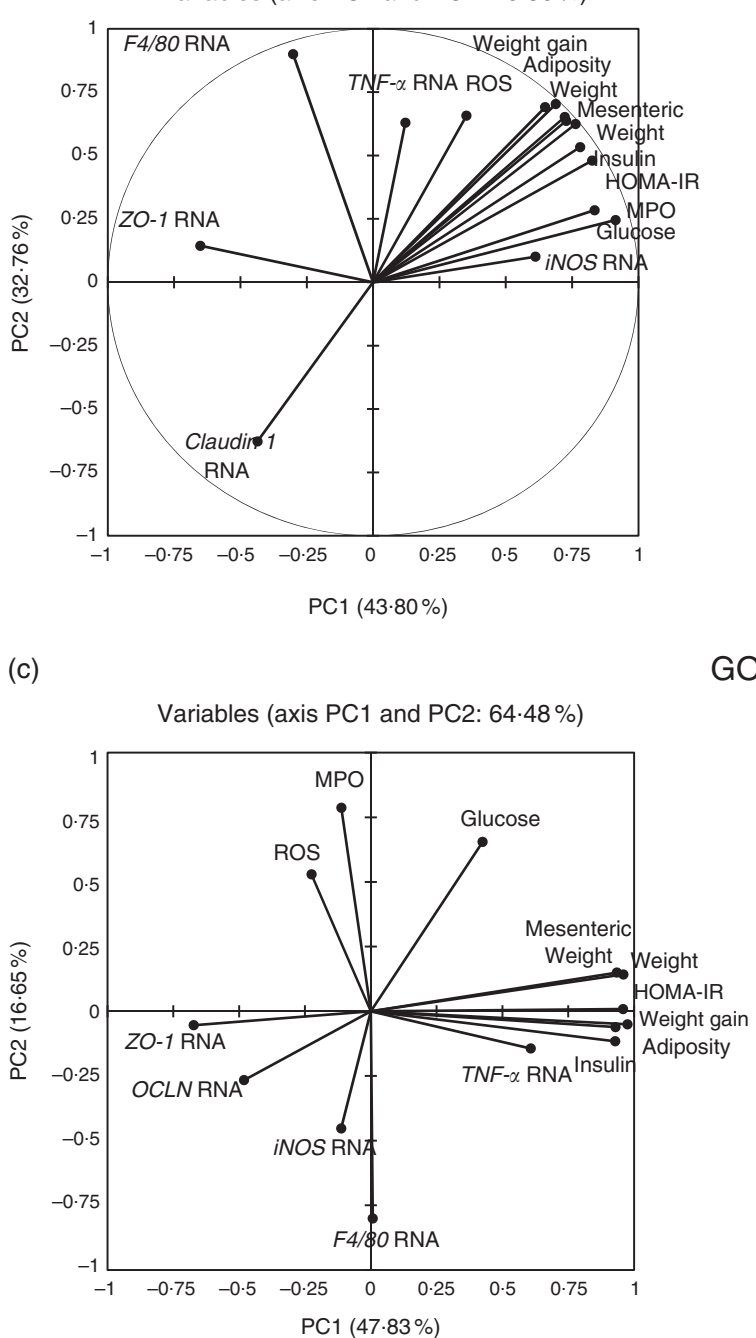

GO
17 weeks

Variables (axis PC1 and PC2: $76 \cdot 56 \%$ )

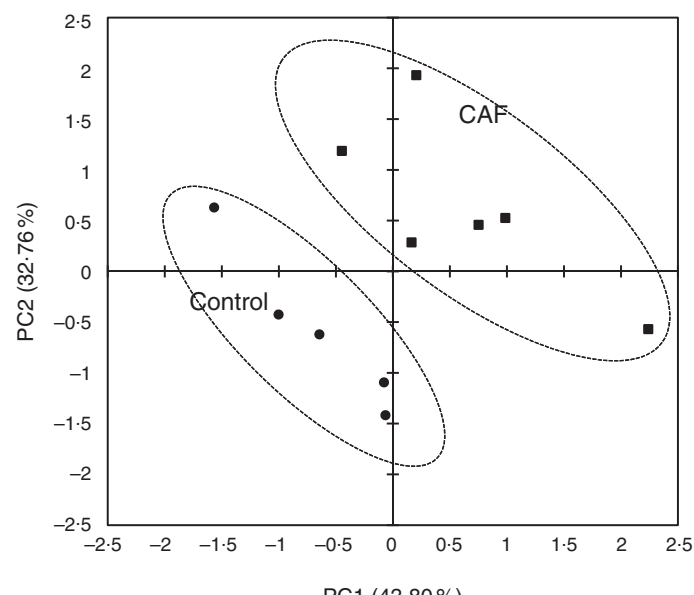

PC1 (43.80\%)

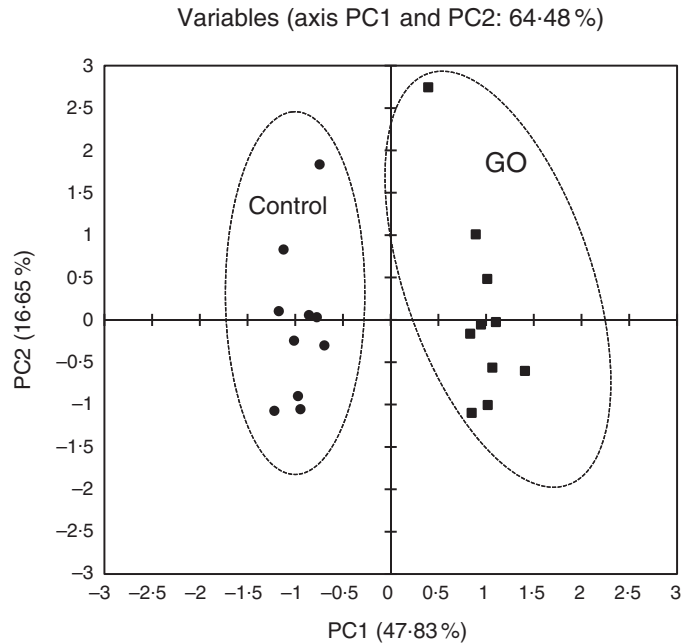

Fig. 4. Principal component analysis (PCA) of all the variables analysed during the different periods of cafeteria or standard diet consumption and in the rat genetic model of obesity. The left panel represents the projection of the variables defined in the plane by the first two principal components (PC), and the right panel shows the PCA score plot of the groups analysed after 14.5 (a) or 17 weeks (b) of CAF intervention or genetic obesity (GO) (c). CAF, cafeteria diet; ROS, reactive oxygen species; MPO, myeloperoxidase; iNOS, inducible nitric oxide synthase; HOMA-IR, homeostasis model assessment of insulin resistance; ZO-1, zonula occludens-1; OCLN, occludin; F4/80, EGF-like module-containing, mucin-like hormone receptor 1. 
neutrophil granulocytes, but this molecule also acts as a proinflammatory mediator and can cause tissue damage during acute or chronic inflammation ${ }^{(32)}$. In this context, upon detecting inflammatory signals, neutrophils change their responsiveness to allow directed migration and enhancement of microbicidal capacity. Activated polymorphonuclear neutrophils are able to synthesise and store enzymes and ROS within cytoplasmic granules $^{(33)}$. The most abundant granule enzyme is MPO, which forms cytotoxic hypochlorous acid from the reaction of chloride anions with the hydrogen peroxide produced after the respiratory burst. Beyond its bactericidal activity, MPO affects various pathways involved in cell signalling and cell-cell interactions, and thus is capable of modulating inflammatory responses ${ }^{(34)}$. Thus, the increase in MPO activity found in correlation with obesity in these animals from the first time point might indicate that inflammation precedes the oxidative stress disturbance induced by diet in the ileum. In agreement with these results, MPO activity in Zucker rats was similar to that in the lean group, a finding that is in concordance with the absence of oxidative stress in this genetic model. This fact supports the hypothesis that the CAF diet might be responsible for the observed changes in intestinal inflammation associated with obesity.

When analysing proinflammatory gene expression after the consumption of the CAF diet, we only found a significant increase in $T N F-\alpha$ levels at the 14.5-week time point; however, the other genes indicating macrophage infiltration and activity, such as F4/80 and $i N O S$, showed a trend towards increased expression. These results again suggest that inflammation is observed from the first time point studied, whereas oxidative stress only becomes apparent at 17 weeks. Furthermore, TNF- $\alpha$ expression was positively associated with measures of adiposity. Importantly, iNOS expression was also correlated with glucose levels. These results are in agreement with the hypothesis that intestinal inflammation might be linked to the development of obesity-associated pathologies ${ }^{(35)}$.

Immune cells, including neutrophils, have been directly implicated in disrupting TJ barrier function. It has been postulated that the proinflammatory cytokine-induced opening of the intestinal TJ barrier is an important mechanism contributing to the TJ barrier defects present in various inflammatory conditions of the gut ${ }^{(36)}$. The same pattern is reflected by the expressions of the permeability genes ZO-1 and claudin-1, whose expressions only decrease significantly after 17 weeks of the CAF diet. Thus, altered permeability is suspected to appear at later stages, concomitant with a high degree of oxidative stress and sustained inflammation. When the correlations between these parameters were analysed, a strong positive association was found between ROS and ileal inflammatory state after 17 weeks of the CAF diet, suggesting a close link between ROS and inflammation. Moreover, the intestinal dysfunction found in the gut (oxidative stress and inflammation) in response to an obesogenic diet was correlated with the degree of obesity and insulin resistance. Taken together, our results suggest that, first, the CAF diet stimulates gut inflammation and, second, in addition to the sustained inflammatory state there is an increase in oxidative stress and relaxation of the permeability barrier. Furthermore, this intestinal dysfunction might be linked to the development of obesity-associated pathologies.
The most surprising results obtained in this study were observed in the genetic model, in which the TJ elements $Z O-1$ and claudin-1 were also underexpressed. Such effects have not been described previously in this animal model. Similarly, previous investigations have demonstrated that leptin increases intestinal permeability ${ }^{(37,38)}$. In particular, the chronic secretion of high levels of leptin by visceral adipose tissue in the context of ageing or obesity permanently impacts the structure and function of the intestinal epithelial barrier by binding LepR on the basolateral side of intestinal epithelial cells and stimulating the RhoA/ ROCK (Ras homolog gene family, member A/rho-kinase) pathway ${ }^{(37)}$. Upon LepR activation, the small GTPase RhoA and its effector ROCK are important modulators of actin cytoskeleton organisation in response to physiological and pathological stimuli $^{(39,40)}$. Disruptions in this pathway have been demonstrated to contribute to obesity and insulin resistance in mice fed a HFD. Despite the fact that obese Zucker rats display markedly elevated circulating leptin levels compared with their lean counterparts, they are LepR deficient, meaning that the changes in intestinal permeability cannot be explained by this mechanism. Taking the fact that these animals were fed standard chow diet into account, the modulation of intestinal permeability through the hyperphagic and obesogenic effects of the CAF diet could also be discarded. Unexpectedly, $T N F-\alpha$ was significantly up-regulated in obese Zucker rats with respect to lean animals. Again, the proinflammatory cytokine-induced opening of the intestinal TJ barrier might be an important mechanism contributing to the TJ barrier defects present in these rats ${ }^{(36)}$; however, additional mechanistic studies are needed to confirm this hypothesis. Overall, the maintenance of intestinal barrier homoeostasis requires complex interactions between host molecules and other environmental factors such as diet, as well as the microbiome. Commensal bacteria have also been proposed as key modulators of intestinal barrier function ${ }^{(41)}$. Other data suggest that certain bacterial species produce metabolites that can influence intestinal permeability and integrity ${ }^{(42)}$. Therefore, it could be possible that obese Zucker rats might have a different microbiome than lean animals, which would explain the changes in the expressions of key genes involved in maintaining intestinal barrier integrity observed in obese animals. However, a study performed in Zucker rats found no significant differences in the intestinal bacterial profiles of the two Zucker rat genotypes at either the phylum or the family level in taxonbased analyses ${ }^{(43)}$. Thus, it is unclear which pathways could be responsible for these changes, and more studies are needed to address this topic in the future.

In summary, in high-fat/high-carbohydrate diet models, the deregulation of gut homoeostasis in response to chronic lipid and carbohydrate overload leads to impaired metabolic homoeostasis, gut inflammation, oxidative stress and changes in intestinal permeability markers in a time-dependent manner. Interventions that limit the intestinal alterations induced by a CAF diet may protect against obesity and insulin resistance.

\section{Acknowledgements}

This study was supported by a grant (AGL2014-55347-R) from the Spanish Ministerio de Economía y Competitividad. K. G.-C. 
and I. G. are student fellows from the Martí i Franquès programme of Rovira $i$ Virgili University. M. P. is a Serra Húnter fellow.

K. G.-C. performed the laboratory work, initiated the literature search and was in charge of drafting the manuscript. Both K. G.-C. and I. G. designed the figures. M. P. and A. A. revised the first drafts. X. T. and M. B. were responsible for final editing and content. All authors critically reviewed the manuscript and approved the final version.

The authors declare that there are no conflicts of interest.

\section{Supplementary material}

For supplementary material/s referred to in this article, please visit https://doi.org/10.1017/S0007114516004608

\section{References}

1. Woods SC, Seeley RJ, Rushing PA, et al. (2003) A controlled high-fat diet induces an obese syndrome in rats. J Nutr $\mathbf{1 3 3}$, 1081-1087.

2. Pranprawit A, Wolber FM, Heyes JA, et al. (2013) Short-term and long-term effects of excessive consumption of saturated fats and/or sucrose on metabolic variables in Sprague Dawley rats: a pilot study. J Sci Food Agric 93, 3191-3197.

3. Savastano DM \& Covasa M (2005) Adaptation to a high-fat diet leads to hyperphagia and diminished sensitivity to cholecystokinin in rats. J Nutr 135, 1953-1959.

4. Fritsche KL (2015) The science of fatty acids and inflammation. Adv. Nutr 6, 293S-301S.

5. Kern PA, Ranganathan S, Li C, et al. (2001) Adipose tissue tumor necrosis factor and interleukin- 6 expression in human obesity and insulin resistance. Am J Physiol Endocrinol Metab 280, E745-E751.

6. Syrenicz A, Garanty-Bogacka B, Syrenicz M, et al. (2006) Low-grade systemic inflammation and the risk of type 2 diabetes in obese children and adolescents. Neuro Endocrinol Lett 27, 453-458.

7. Brake DK, Smith EO, Mersmann H, et al. (2006) ICAM-1 expression in adipose tissue: effects of diet-induced obesity in mice. Am J Physiol Cell Physiol 291, C1232-C1239.

8. Xu H, Barnes GT, Yang Q, et al. (2003) Chronic inflammation in fat plays a crucial role in the development of obesity-related insulin resistance. J Clin Invest 112, 1821-1830.

9. Kiela PR \& Ghishan FK (2016) Physiology of intestinal absorption and secretion. Best Pract Res Clin Gastroenterol 30, $145-159$.

10. Worthington JJ (2015) The intestinal immunoendocrine axis: novel cross-talk between enteroendocrine cells and the immune system during infection and inflammatory disease. Biochem Soc Trans 43, 727-733.

11. de La Serre CB, Ellis CL, Lee J, et al. (2010) Propensity to highfat diet-induced obesity in rats is associated with changes in the gut microbiota and gut inflammation. Am J Physiol Gastrointest Liver Physiol 299, G440-G448.

12. Sies H, Stahl W \& Sevanian A (2005) Nutritional, dietary and postprandial oxidative stress. J Nutr 135, 969-972.

13. Halliwell B, Zhao K \& Whiteman M (2000) The gastrointestinal tract: a major site of antioxidant action? Free Radic Res 33, 819-830.

14. Toumi R, Soufli I, Rafa H, et al. (2014) Probiotic bacteria lactobacillus and bifidobacterium attenuate inflammation in dextran sulfate sodium-induced experimental colitis in mice. Int J Immunopathol Pharmacol 27, 615-627.

15. Wang W, Xia T \& Yu X (2015) Wogonin suppresses inflammatory response and maintains intestinal barrier function via TLR4-MyD88-TAK1-mediated NF-кB pathway in vitro. Inflamm Res 64, 423-431.

16. Kolios G, Valatas V \& Ward SG (2004) Nitric oxide in inflammatory bowel disease: a universal messenger in an unsolved puzzle. Immunology 113, 427-437.

17. Oshima T \& Miwa H (2016) Gastrointestinal mucosal barrier function and diseases. J Gastroenterol 51, 768-778.

18. Luo H, Guo P \& Zhou Q (2012) Role of TLR4/NF-kappaB in damage to intestinal mucosa barrier function and bacterial translocation in rats exposed to hypoxia. PLOS ONE 7, e46291.

19. Bruewer M, Luegering A, Kucharzik T, et al. (2003) Proinflammatory cytokines disrupt epithelial barrier function by apoptosis-independent mechanisms. J Immunol 171, 6164-6172.

20. Katz KD, Hollander D, Vadheim CM, et al. (1989) Intestinal permeability in patients with Crohn's disease and their healthy relatives. Gastroenterology 97, 927-931.

21. Barrenetxe J, Villaro AC, Guembe L, et al. (2002) Distribution of the long leptin receptor isoform in brush border, basolateral membrane, and cytoplasm of enterocytes. Gut 50, 797-802.

22. El Homsi M, Ducroc R, Claustre J, et al. (2007) Leptin modulates the expression of secreted and membrane-associated mucins in colonic epithelial cells by targeting PKC, PI3K, and MAPK pathways. Am J Physiol Gastrointest Liver Physiol 293, G365-G373.

23. Morris DL \& Rui L (2009) Recent advances in understanding leptin signaling and leptin resistance. Am J Physiol Endocrinol Metab 297, E1247-E1259.

24. Baselga-Escudero L, Pascual-Serrano A, Ribas-Latre A, et al. (2015) Long-term supplementation with a low dose of proanthocyanidins normalized liver miR-33a and miR-122 levels in high-fat diet-induced obese rats. Nutr Res 35, 337-345.

25. Aleixandre de Artinano A \& Miguel Castro M (2009) Experimental rat models to study the metabolic syndrome. Br J Nutr 102, 1246-1253.

26. Mizuno M, Sada T, Kato M, et al. (2002) Renoprotective effects of blockade of angiotensin II AT1 receptors in an animal model of type 2 diabetes. Hypertens Res 25, 271-278.

27. van den Brom CE, Huisman MC, Vlasblom R, et al. (2009) Altered myocardial substrate metabolism is associated with myocardial dysfunction in early diabetic cardiomyopathy in rats: studies using positron emission tomography. Cardiovasc Diabetol 8, 1-12.

28. Kaur J (2014) A comprehensive review on metabolic syndrome. Cardiol Res Pract 2014, 943162.

29. Savini I, Catani MV, Evangelista D, et al. (2013) Obesityassociated oxidative stress: strategies finalized to improve redox state. Int J Mol Sci 14, 10497-10538.

30. Codoñer-Franch $\mathrm{P}$, Tavárez-Alonso $\mathrm{S}$, Murria-Estal $\mathrm{R}$, et al. (2012) Elevated advanced oxidation protein products (AOPPs) indicate metabolic risk in severely obese children. Nutr Metab Cardiovasc Dis 22, 237-243.

31. Dandona P, Ghanim H, Chaudhuri A, et al. (2010) Macronutrient intake induces oxidative and inflammatory stress: potential relevance to atherosclerosis and insulin resistance. Exp Mol Med 42, 245-253.

32. Davies MJ (2011) Myeloperoxidase-derived oxidation: mechanisms of biological damage and its prevention. J Clin Biochem Nutr 48, 8-19.

33. Mariani F, Sena P \& Roncucci L (2014) Inflammatory pathways in the early steps of colorectal cancer development. World J Gastroenterol 20, 9716-9731.

34. van der Veen BS, de Winther MPJ \& Heeringa P (2009) Myeloperoxidase: molecular mechanisms of action and 
their relevance to human health and disease. Antioxid Redox Signal 11, 2899-2937.

35. Ding S, Chi MM, Scull BP, et al. (2010) High-fat diet: bacteria interactions promote intestinal inflammation which precedes and correlates with obesity and insulin resistance in mouse. PLOS ONE 5, e12191.

36. Suzuki T (2013) Regulation of intestinal epithelial permeability by tight junctions. Cell Mol Life Sci 70, 631-659.

37. Le Dréan G, Haure-Mirande V, Ferrier L, et al. (2014) Visceral adipose tissue and leptin increase colonic epithelial tight junction permeability via a RhoA-ROCK-dependent pathway. FASEB J 28, 1059-1070.

38. Kim CY \& Kim KH (2014) Curcumin prevents leptin-induced tight junction dysfunction in intestinal Caco-2 BBe cells. J Nutr Biochem 25, 26-35.
39. Ivanov AI, Parkos CA \& Nusrat A (2010) Cytoskeletal regulation of epithelial barrier function during inflammation. $A m J$ Pathol 177, 512-524.

40. Citalán-Madrid AF, García-Ponce A, Vargas-Robles $\mathrm{H}$, et al. (2013) Small GTPases of the Ras superfamily regulate intestinal epithelial homeostasis and barrier function via common and unique mechanisms. Tissue Barriers 1, e26938.

41. Hooper LV \& Gordon JI (2001) Commensal host-bacterial relationships in the gut. Science 292, 1115-1118.

42. Natividad JMM \& Verdu EF (2013) Modulation of intestinal barrier by intestinal microbiota: pathological and therapeutic implications. Pharmacol Res 69, 42-51.

43. Lees H, Swann J, Poucher SM, et al. (2014) Age and microenvironment outweigh genetic influence on the zucker rat microbiome. PLOS ONE 9, e100916. 